\title{
Heart Failure with Reduced Ejection Fraction, Multiple-Valve Diseases, And Left Atrial Thrombus: A Case Report of Determining Whether to Do Intervention Strategies or Conservative Medical Therapies Only
}

\author{
Hasan M.R.D. ${ }^{1}$, Andrianto A. ${ }^{1}$, Subagjo A. ${ }^{1 *}$ \\ andrianto@fk.unair.ac.id \\ ${ }^{1}$ Department of Cardiology and Vascular Medicine, Universitas Airlangga, Surabaya, 60286, Indonesia
}

\begin{abstract}
Structural valvular heart disease (VHD) may be the cause of heart failure or may worsen the clinical status of patients with heart failure. Heart failure patients with VHD are at increased risk of events including sudden cardiac death. Before considering intervention (surgical or percutaneous) all patients should receive appropriate medical therapy. Numerous percutaneous and/or hybrid procedures have been introduced in the past few years and they are changing the management of VHD. In patients with heart failure and valvular heart disease, either primary or functional, the whole process of decision-making should be staged through a comprehensive evaluation of the risk-benefit ratio of different treatment strategies. In this case, a heart failure patient with multiple-valve diseases, LA thrombus, and dilated cardiomyopathy was referred to a faraway cardiac center to undergo intervention procedures. This is a case report of a 59-yo female who presented to the emergency ward, she was referred with the diagnosis of Chronic Heart Failure due to multiple-valve diseases, atrial fibrillation, hypo-potassium, and low blood pressure. From echocardiography, we found severe mitral stenosis, severe aortic stenosis, moderate aortic insufficiency, moderate tricuspid stenosis, severe tricuspid insufficiency, mild pulmonic insufficiency, with left atrial thrombus, and dilated cardiomyopathy. However, after the evaluation was made and had a discussion with the patient about the risk-benefit of intervention procedures, the patient chose to not undergoing the intervention procedures even though there was no contraindication found in the patients to the procedures that should be done to the patient.
\end{abstract}

Keywords: Heart failure; multiple-valve diseases; left atrial thrombus; intervention procedure option

\section{Introduction}

Structural VHD can be a cause of heart failure or can worsen the clinical status of patients with heart failure. Heart failure can also occur in patients who have had valve surgery. Patients with heart failure with VHD are at increased risk for cardiac events including sudden cardiac death (Falk et al., 2017). Before 
considering any intervention (surgical or percutaneous) all patients should receive appropriate medical. Many percutaneous and/or hybrid procedures have been introduced in recent years and they are changing the management of VHD (Rosano, 2017). In patients with heart failure and VHD, whether primary or functional, the entire decision-making process should be carried out through a comprehensive evaluation of the riskbenefit ratio of various treatment strategies (Gong et al., 2018; Khan et al., 2020). In addition, good education and a well-planned referral system for further action can help reduce the costs involved in treating patients (Baumgartner et al., 2017a; Reyes et al., 2016).

We present a case with multiple-valve diseases that has progressed to heart failure with reduced ejection fraction and LA thrombus that was sent from a referring hospital in the hope of implementing an intervention strategy. The purpose of this case report is to consider all existing valve diseases in determining intervention strategies for multiple-valve diseases (not specifying intervention strategies individually on each valve) and emphasizes the importance of educating patients about disease diagnosis, recommendations for intervention strategies and medical therapy. to be given, the risks-benefits of the intervention, and the prognosis of the patient's disease. These things will help doctors and patients in determining the treatment to be taken next.

\section{Case}

A 59-year-old woman came to the emergency room after getting referred by a faraway hospital for further treatment for Chronic Heart Failure due to multiple-valve diseases, atrial fibrillation, hypo-potassium, and low blood pressure. Her chief complaint was shortness of breath, especially after traveling by plane recently. The patient cannot sleep on a flatbed. The patient had just been discharged from the referral hospital after 23 days of treatment due to acute decompensated heart failure. Her history of multiple-valve diseases has been known in the last 20 years, with the also the history of undergone balloon mitral valvuloplasty (BMV) procedure performed at a heart center hospital. There is no history of previous illness in the patient. So far, she has been seeking treatment from a cardiologist at a public hospital in the city where the patient lives.

From the physical examination, the patient found conjunctival anemia, increased JVP. Auscultation revealed irregular heart sounds, grade III/IV diastolic murmur at the apex, grade III/VI systolic murmur at the lower left sternal border, minimal lung rales on both sides, and no swollen lower limbs. From the chest XRay, cardiomegaly (CTR 60\%), mitral heart configuration, and emphysematous lung were obtained. From the ECG, the picture shows atrial fibrillation slow ventricular response 60x/min, normoaxis, slow progression of R V1-V3 (Figure 1).
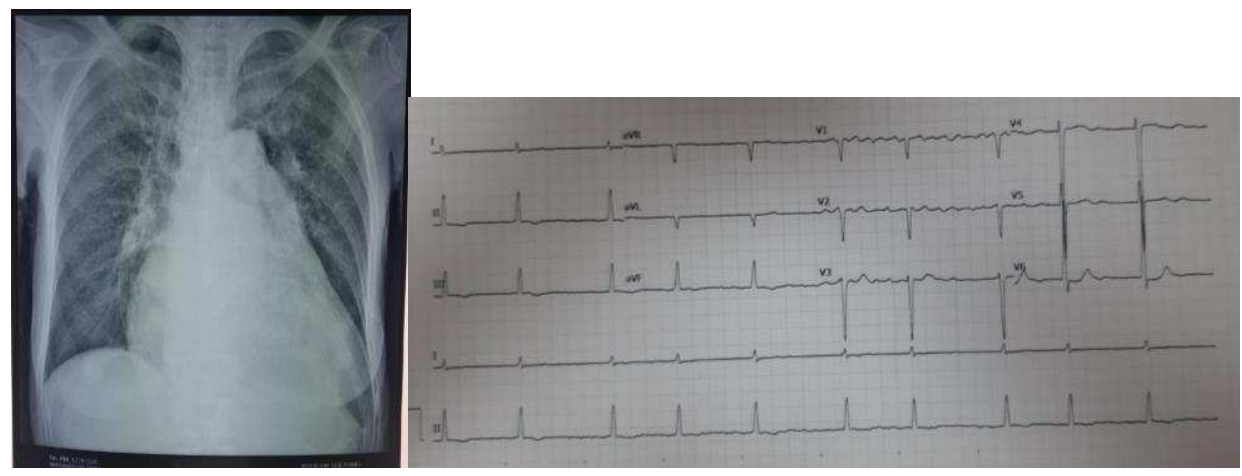

Figure 1. patient chest $\mathrm{x}$-ray (left) and patient ECG (right) 
Echocardiography examination revealed severe mitral stenosis [mitral valve (MV) Mean pressure gradient (PG) $10.33 \mathrm{mmHg}$; mitral valve area (MVA) Planimetry $0.6 \mathrm{~cm} 2$ ] Wilkins score 2-3-2-3; severe aortic stenosis (AS) [aortic valve area (AVA) planimetry $0.6 \mathrm{~cm} 2$; AV mean PG $39.72 \mathrm{mmHg}$; moderate aortic regurgitation (AR) [AR deceleration slope $2.9 \mathrm{~m} / \mathrm{s} 2$; AR pressure half-time (PHT) 451 ms]; moderate tricuspid stenosis (TS) [tricuspid valve (TV) mean PG $2.51 \mathrm{mmHg}$ ); severe tricuspid regurgitation (TR) (TR max PG $101.72 \mathrm{mmHg}$ ); mild pulmonic regurgitation (PR deceleration slope $14 \mathrm{~m} / \mathrm{s} 2$ ); severe pulmonic hypertension (PHT) [estimated pulmonary artery systolic pressure (PASP) $111 \mathrm{mmHg}$; pulmonic valve (PV) acceleration time (AccT) $55 \mathrm{~ms}$; ; all cardiac chamber revealed dilated [left ventricular internal diameter end diastole (LVIDD) 5,1 cm]; reduced left ventricular (LV) systolic function [ejection fraction (EF) by teich $33 \%$; EF by biplane 33\%]; right ventricular (RV) also show reduced systolic function (Tapse $1.3 \mathrm{Cm}$ ); LV segmental analysis show global hypokinetic with eccentric left ventricular hypertrophy (LVH); there was also show thrombus in LA with the size of $2.9 \mathrm{x} 4.7 \mathrm{Cm}$; no dilatation of aortic and aortic root was shown (Figure 2).

From the examination results, it was concluded that the patient was diagnosed with chronic heart failure; rheumatic heart disease (RHD) severe MS - severe AS - moderate AR - moderate TS - severe TR - mild PR; severe PHT; left atrial thrombus; atrial fibrillation moderate ventricular response; and dilated cardiomyopathy. Then the patient was informed about her diagnosis, the intervention that procedures that should be done to the patient, which are: MVR; AVR; tricuspid annuloplasty; LA thrombus evacuation; CABG procedure if required depending on the result of diagnostic coronary angiography (DCA); and also, about the risk and benefit of the procedure that will be carried out. After consultation, the patient chose not to undergo any of those intervention procedures.

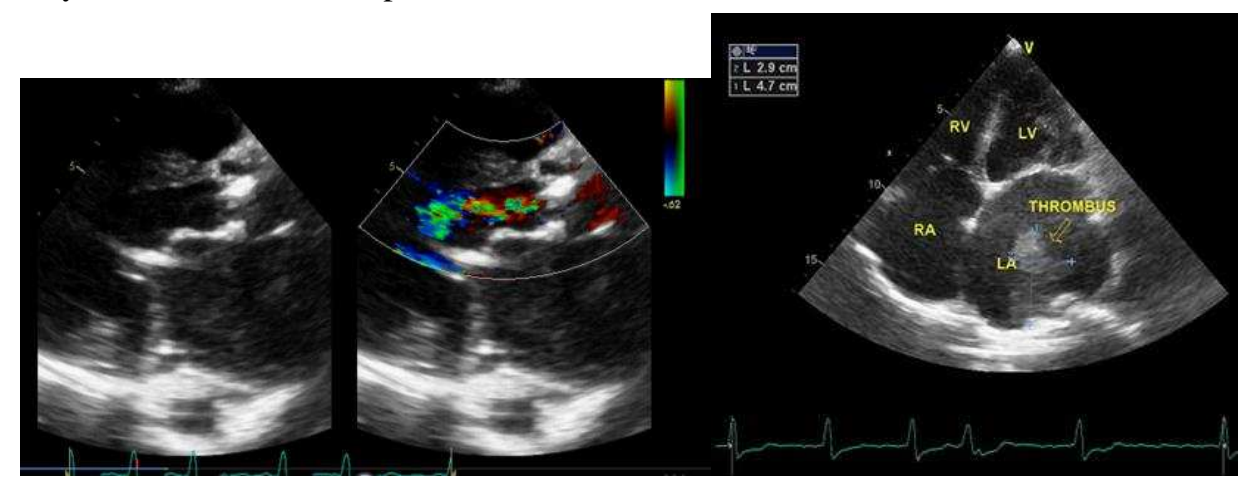

Figure 2. Parasternal long-axis view showing calcification of mitral and aortic valves with aortic regurgitation jet (left) and apical-fourchamber view showing all chamber dilatation with LA thrombus (right)

\section{Discussion}

In determining what intervention procedures can be performed on the patient, whether the intervention is minimally invasive or surgical, or the patient is only receiving medical therapy without invasive strategy procedures, we refer to the Guidelines for the management of VHD from the European Society of Cardiology (ESC). In the guidelines, the only contraindication to do intervention in patients with VHD is patients with severe comorbidities in which intervention is unlikely to improve quality or survival, in which no such contraindication was found in this case (Baumgartner et al., 2017a).

In this patient, we have multiple-valve diseases and combinations of stenosis and regurgitation. There are five general principles for the management of combined or multiple-valve diseases which are: The first is that 
when regurgitation or stenosis is the main VHD, management should follow the recommendations for that VHD. When the severity of regurgitation and stenosis is balanced, treatments should be based on objective symptoms and consequences rather than regurgitation or stenosis severity index. In this case, the pressure gradient must be taken into account rather than the valve area or regurgitation measurements for determining the severity of the condition. (Baumgartner et al., 2017a).

The second is that, in addition to assessing each valve lesion separately, it is also required to consider the interactions between the various valve lesions. As an illustration, concomitant mitral regurgitation can contribute to an overestimate of the severity of aortic stenosis because mitral regurgitation limits flow across the aortic valve, lowering the aortic gradient. This emphasizes the importance of combining different data, including valve area evaluation, utilizing approaches that are less dependent on loading circumstances, such as planimetry, if possible. (Baumgartner et al., 2017a).

The third principle is that interventional indications are based on a broad assessment of the effects of various valvular abnormalities (i.e., symptoms or presence of LV dilatation or dysfunction). Interventions may be undertaken in the case of non-severe multiple lesions that cause symptoms or compromise LV function. Fourth, the presence of other VHDs must be considered when selecting a surgical procedure; valve repair remains the best option (Baumgartner et al., 2017a).

Fifth, the decision to intervene on multiple valves must take into account the additional surgical risks of the combined procedure (Baumgartner et al., 2017a). Patients that undergoing open-heart surgery usually will be using a heart-lung machine. Although it is an amazing machine, the heart-lung machine had many adverse effects that can occur. A systemic inflammatory response is the most common due to the blood being introduced to an entirely new environment. This inflammatory response can lead to many other problems throughout the body and ultimately organ failure may occur. Other adverse effects are atrial fibrillation, pulmonary damage including edema and ischemia, renal failure, and neurocognitive deficits. While some of these are not common effects, research has shown that they certainly are possible and harmful (Steidl, 2011; Tamargo et al., 2020).

Besides the adverse effects of the heart-lung machine, there are also adverse effects of general anesthesia that should be considered. Most short-term adverse effects occur immediately and last only for a short while. The most common that occur are feeling confused. Though rarely occurs, sometimes the patient feels nausea and vomiting, dry mouth, sore throat or hoarseness, chills and shivering because of dropped body temperature, dizziness, and bladder problems. Long-term adverse effects that could occur are Postoperative delirium that could last for a week and Postoperative cognitive dysfunction (POCD). POCD is more likely to develop the longer the patient undergoing general anesthesia and if the patient had a history of stroke, heart disease, lung disease, Alzheimer's disease, and Parkinson's disease (Chen et al., 2020; Damshenas and Kalani, 2016).

\subsection{Mitral Valve Stenosis}

The patient fits the indication criteria for percutaneous mitral commissurotomy (PMC) in the selection of interventional mitral stenosis in patients. In general, intervention should be reserved for individuals with clinically significant mitral stenosis (moderate to severe, and MVA $<1.5 \mathrm{~cm} 2$ ). However, if symptoms cannot be explained by other causes and the anatomy is good, PMC may be considered in symptomatic individuals with valve area $>1.5 \mathrm{~cm} 2$. (Baumgartner et al., 2017a).

However, although the patient met the criteria for PMC, from the examination of the patient, we found contraindications for PMC, namely the presence of a thrombus in the left atrial and the presence of severe aortic valve disease and a combination of stenosis and regurgitation of the tricuspid valve that requires surgery. So that the intervention that can be performed on the patient is mitral valve surgery according to the 
ESC algorithm (see Figure 3). The most important contraindication for PMC is LA thrombus. However, when the thrombus is located in the LA appendage, PMC may be considered in patients without an urgent need for intervention, provided repeated echocardiography shows that the thrombus has disappeared after 1-3 months of oral anticoagulation. Surgery is indicated if the thrombus persists. In asymptomatic mitral stenosis patients, surgery is performed in patients at high risk of cardiac complications who have contraindications to PMC and are at low risk for surgery (Baumgartner et al., 2017a).

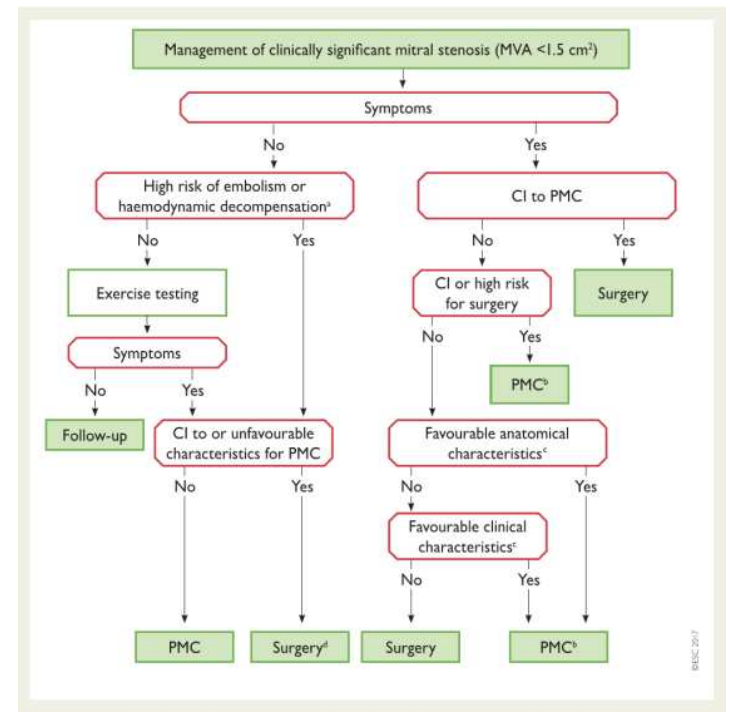

Figure 3. Management of severe mitral stenosis clinically significant (MVA $<1.5 \mathrm{~cm} 2)$ (Baumgartner et al., 2017a)

\subsection{Aortic Valve Stenosis and Regurgitation}

The main diagnostic tool is echocardiography. This validates aortic stenosis, evaluates valve calcification, LV function, and wall thickness, detects the existence of additional related valvular disease or aortic pathology, and offers prognostic information. When it comes to determining the severity of aortic stenosis, Doppler echocardiography is the preferred method. Although the valve area appears to be an appropriate measure for determining the severity of aortic stenosis from a theoretical standpoint, it has technical limitations in clinical practice. Overall flow rate, mean pressure gradient (the strongest parameter), ventricular function, wall size and thickness, degree of valvular calcification, blood pressure, and functional status should all be considered when making clinical decisions. When hypertensive individuals become normotensive, they should be evaluated. (Baumgartner et al., 2017b).

The results of the examination showed a patient with symptomatic severe aortic stenosis and moderate aortic regurgitation. Echocardiographic examination showed that the patient was in the category of low-flow (Svi $29.42 \mathrm{ml} / \mathrm{m} 2$ ), low-gradient (AV mean PG $39.72 \mathrm{mmHg}$ ) aortic stenosis with reduced ejection fraction (EF by Teich $31 \%$; by biplane 33\%). The next step should be to do a dobutamine stress echocardiography, to see if the patient still has a flow reserve. In patients with low-flow, low-gradient aortic stenosis with reduced ejection fraction where the decrease in ejection fraction is largely due to excessive afterload, LV function usually improves after the intervention. In contrast, improvement in LV function after the intervention is uncertain if the underlying cause is scarring due to extensive myocardial infarction or cardiomyopathy. Interventions are advised to be performed when severe aortic stenosis is present with the increased flow (true 
severe aortic stenosis)(Baumgartner et al., 2017a) While patients classified as having pseudosevere aortic stenosis on increased flow should receive conventional treatment for heart failure(Annabi M et al., 2019) Although the outcome of this intervention in patients without reserve flow is compromised by higher operative mortality, surgical aortic valve replacement (SAVR), as well as transcatheter aortic valve implantation (TAVI), has also been shown to improve ejection fraction and clinical status in these patient (Sergi et al., 2019).

\subsection{Tricuspid Valve Stenosis and Regurgitation}

Tricuspid stenosis is frequently associated with tricuspid regurgitation, which is most commonly due to rheumatic causes. As a result, it's almost always linked to left-sided valve diseases, particularly mitral stenosis, which frequently takes center stage in the clinical picture. Congenital, drug-induced valve illness, Whipple's disease, endocarditis, and large right atrial tumors are among the less common causes. (Baumgartner et al., 2017a)

While the pathology of tricuspid regurgitation is more often secondary, it is caused by RV dysfunction as a result of pressure and/or volume overload on a structurally normal valve. Infective endocarditis (especially in intravenous drug addicts), rheumatic heart disease, carcinoid syndrome, myxomatous disease, endomyocardial fibrosis, Ebstein's anomaly and congenital dysplastic valves, drug-induced valvular disease, thoracic valve trauma, and iatrogenic damage are all possible causes of primary tricuspid regurgitation (Baumgartner et al., 2017a)

The most useful information comes from echocardiography. Tricuspid stenosis is frequently ignored and necessitates a thorough examination. Valve reparability is determined by an echocardiographic examination of the valve's anatomy and its subvalvular apparatus. The origin of primary tricuspid regurgitation is usually determined by a specific abnormality of the valve anatomy. The degree of dilatation of the annulus, the size and function of the RV, and the degree of distortion of the tricuspid valve should all be measured in secondary tricuspid regurgitation. The severity of tricuspid regurgitation should be assessed (using a combination of qualitative and quantitative indicators) as well as the pulmonary systolic pressure. It should be noted that the existence of severe tricuspid regurgitation may disguise the problem of increased pulmonary vascular resistance because the rate may be lower than predicted in the case of pulmonary hypertension (Baumgartner et al., 2017a)

Catheterization is no longer used to diagnose or assess the severity of tricuspid stenosis and tricuspid regurgitation, but it should be done in patients who are having isolated tricuspid valve surgery with secondary tricuspid regurgitation to assess hemodynamics, particularly pulmonary vascular resistance (Baumgartner et al., 2017a).

In this patient's case, we found a patient with moderate tricuspid valvular disease (TV mean PG 2.51 $\mathrm{mmHg}$ ) and severe tricuspid regurgitation (TR max PG $101.72 \mathrm{mmHg}$ ). In patients with severe tricuspid regurgitation, both primary and secondary causes who will undergo surgical intervention on the left heart valve, it is recommended to undergo surgical intervention on the patient's tricuspid valve.

Based on the ESC valve disease management guidelines, the patient is recommended surgical intervention, the patient must undergo 4-5 procedures, namely: mitral valve replacement (Recommendation I/C); surgical aortic valve replacement (Recommendation I/C or IIa/C depending on the results of Dobutamine Echo and CT Calcium Score if needed); surgical tricuspid replacement (Recommendation I/C); thrombectomy for thrombus in LA; and CABG (Recommendation I/C if indicated, according to results of coronary stenosis from DCA examination)(Baumgartner et al., 2017a).

In predicting mortality during hospitalization after surgery, EuroScore II can be used. In a study comparing EuroScore II with the Society of Thoracic Surgeons (STS) Risk Score in cardiac surgery, EuroSCORE II had 
better predictive discrimination for operative mortality than EuroSCORE I, which greatly overestimated this risk. EuroSCORE II also outperformed the STS risk score. The inclusive nature of EuroSCORE II for multiple procedures provides more flexibility than the STS score for complex procedures. EuroSCORE II should be considered to calculate the risk score for complex cardiac surgery patients (Ad et al., 2016) Prediction of mortality during hospitalization after surgery in patients based on Euroscore II is $4.13 \%$.

The next step for the patient is to educate the patient and family about matters related to the patient's illness; prognosis; intervention actions that should be taken; existing procedural problems at the local heart center such as the unavailability of prosthetic tricuspid valve supplies; several more examinations that must be carried out by patients related to determining the prognosis of surgical intervention (dobutamine echo, CT cardiac / DCA) so that the risk-benefit of surgery can be determined. After education, the patient decides to refuse the currently recommended intervention.

\section{Conclusion}

Structural valvular heart disease can be a cause of heart failure or can worsen the clinical status of patients with heart failure. Heart failure can also occur in patients who have had valve surgery. The general principle for the management of combined or multiple-valve diseases is that in combined VHD, valvular pathology is considered severe even if the stenosis and regurgitation are only of moderate severity and the pressure gradient is critical for assessment; and management of multiple-valve diseases is determined by the predominant VHD. Coronary evaluation using DCA/CT is recommended for severe VHD patients who will undergo intervention and have cardiovascular risk factors. Education to patients about understanding the patient's disease, recommendations for intervention and treatment to be carried out, the risk-benefit of the intervention, and the prognosis of the disease will help patients and doctors in making decisions about the next treatment.

\section{References}

Ad, N., Holmes, S.D., Patel, J., Pritchard, G., Shuman, D.J., Halpin, L., 2016. Comparison of EuroSCORE II, Original EuroSCORE, and The Society of Thoracic Surgeons Risk Score in Cardiac Surgery Patients. Annals of Thoracic Surgery.

Annabi, M.S., Dahou, A., Burwash, I.G., Bartko, P.E., Bergler-Klein, J., Mascherbauer, J., Mundigler, G., Orwat, S., Baumgartner, H., Cavalcante, J., Ribeiro, H.B., Rodes-Cabau, J., Clavel, M.A., Pibarot, P., 2019. Aortic Valve Replacement is Superior to Conservative Management in Low-Flow, Low-Gradient Aortic Stenosis independently of the presence of true severe stenosis. Archives of Cardiovascular Diseases Supplements 11.

Baumgartner, H., Falk, V., Bax, J.J., De Bonis, M., Hamm, C., Holm, P.J., Iung, B., Lancellotti, P., Lansac, E., Muñoz, D.R., Rosenhek, R., Sjögren, J., Tornos Mas, P., Vahanian, A., Walther, T., Wendler, O., Windecker, S., Zamorano, J.L., Roffi, M., Alfieri, O., Agewall, S., Ahlsson, A., Barbato, E., Bueno, H., Collet, J.P., Coman, I.M., Czerny, M., Delgado, V., Fitzsimons, D., Folliguet, T., Gaemperli, O., Habib, G., Harringer, W., Haude, M., Hindricks, G., Katus, H.A., Knuuti, J., Kolh, P., Leclercq, C., McDonagh, T.A., Piepoli, M.F., Pierard, L.A., Ponikowski, P., Rosano, G.M.C., Ruschitzka, F., Shlyakhto, E., Simpson, I.A., Sousa-Uva, M., Stepinska, J., Tarantini, G., Tche, D., Aboyans, V., 2017a. 2017 ESC/EACTS Guidelines for the management of valvular heart disease. European Heart Journal 38, 2739-2786.

Baumgartner, H., Hung, J., Bermejo, J., Chambers, J.B., Edvardsen, T., Goldstein, S., Lancellotti, P., Lefevre, M., Miller, F., Otto, C.M., 2017b. Recommendations on the echocardiographic assessment of aortic valve stenosis: A focused update from the European Association of Cardiovascular Imaging and the American Society of Echocardiography. European Heart Journal Cardiovascular Imaging.

Chen, W., Yuan, L., Wang, Y., Tang, W., Wang, L., Wang, J., Li, X., Yong, Y., Fu, G., Song, J., 2020. Monitored Anesthesia Care Versus Intubated General Anesthesia for Open Heart Surgery Under Cardiopulmonary Bypass: A Prospective Cohort Study. SSRN Electronic Journal.

Damshenas, M.H., Kalani, N., 2016. Determining retelling of events during general anesthesia in open-heart surgery patients in the hospitals affiliated to shiraz university of medical sciences. IIOAB Journal 7.

Falk, V., Baumgartner, H., Bax, J.J., De Bonis, M., Hamm, C., Holm, P.J., Iung, B., Lancellotti, P., Lansac, E., Muñoz, D.R., Rosenhek, R., Sjögren, J., Tornos Mas, P., Vahanian, A., Walther, T., Wendler, O., Windecker, S., Zamorano, J.L., 2017. 2017 ESC/EACTS Guidelines for the management of valvular heart disease. European journal of cardio-thoracic surgery: official journal of the European Association for Cardio-thoracic Surgery. 
Gong, F.F., Jelinek, M. v., Castro, J.M., Coller, J.M., McGrady, M., Boffa, U., Shiel, L., Liew, D., Wolfe, R., Stewart, S., Owen, A.J., Krum, H., Reid, C.M., Prior, D.L., Campbell, D.J., 2018. Risk factors for incident heart failure with preserved or reduced ejection fraction, and valvular heart failure, in a community-based cohort. Open Heart 5.

Khan, F., Okuno, T., Malebranche, D., Lanz, J., Praz, F., Stortecky, S., Windecker, S., Pilgrim, T., 2020. Transcatheter Aortic Valve Replacement in Patients With Multivalvular Heart Disease. JACC: Cardiovascular Interventions.

Reyes, E.B., Ha, J.W., Firdaus, I., Ghazi, A.M., Phrommintikul, A., Sim, D., Vu, Q.N., Siu, C.W., Yin, W.H., Cowie, M.R., 2016. Heart failure across Asia: Same healthcare burden but differences in organization of care. International Journal of Cardiology.

Rosano, G., 2017. Valvular Heart Disease in Heart Failure. International Cardiovascular Forum Journal.

Sergi, D., Acconcia, M.C., Muscoli, S., Perrone, M.A., Cammalleri, V., di Luozzo, M., Marchei, M., Giannoni, M.F., Barillà, F., Gaudio, C., Chiocchi, M., Romeo, F., Caretta, Q., 2019. Meta-analysis of the impact on early and late mortality of TAVI compared to surgical aortic valve replacement in high and low-intermediate surgical risk patients. European Review for Medical and Pharmacological Sciences.

Steidl, S., 2011. The Adverse Effects of the Cardiopulmonary Bypass Machine. A Senior Thesis.

Tamargo, C.L., Botros, M., Saveanu, R. v., 2020. The relationship between neurocognitive decline and the heart-lung machine. Journal of Cardiac Surgery. 